\title{
Responsabilidad social empresarial de los estudios contables de la ciudad de Pilar
}

\author{
Norimir Primitiva Torres Pedrozo ${ }^{1}$ \\ torresnorimir@gmail.com \\ Tutor metodológico: Jorge Hipólito Cabañas Pereira \\ Jor.caba@hotmail.com \\ Facultad de Ciencias Contables, Administrativas y Económicas \\ Universidad Nacional De Pilar \\ Pilar, Paraguay
}

\section{RESUMEN}

La crisis social, ambiental y económica demanda que el contador púbico se involucre en las acciones de RSE. La investigación describe las prácticas de responsabilidad social empresarial que implementa los contadores públicos de la ciudad de Pilar. Situación que se sustenta en la teoría de Carroll (1999), que construye un modelo de RSC compuesto por cuatro dimensiones: la dimensión económica, la dimensión legal, la dimensión ética y la dimensión filantrópica. De esta base se definen las categorías de análisis que direccionan el estudio. Se utilizará una metodología de nivel descriptivo, observacional con enfoque únicamente cualitativo y de acuerdo al tiempo que se desarrollará la investigación, es de corte trasversal prospectivo. La población está compuesta por 12 estudios contables, legamente establecida en la Municipalidad de Pilar. Como técnica de recolección de datos se utilizará la guía de autoevaluación, tratándose, por lo tanto, de un cuestionario autoadministrado con respuestas binarias (si/no) y comentarios sobre las preguntas planteadas. El cuestionario corresponde a una guía de auto aplicación desarrollado por ADEC-ETHOS denominado indicadores de RSE 1.0. validado en Paraguay por la ADEC. Los resultados hallan incipientes iniciativas no planificadas ni establecidas como políticas organizacionales de los estudios contables, lo que requiere de un abordaje investigación -acción para revertir esta situación hacia una cultura más socialmente responsable con los clientes, la comunidad y consigo misma como organización.

Palabras Clave: responsabilidad social; empresarial; estudios contables.

\footnotetext{
${ }^{1}$ Candidata a Contadora Pública Nacional.
} 
Torres Pedrozo y Cabañas Pereira...

\title{
Corporate social responsibility of the accounting firms of the city of Pilar
}

\begin{abstract}
ABSTRAC
The social, environmental and economic crisis demands that the public accountant get involved in CSR actions. The research describes the corporate social responsibility practices implemented by public accountants in the city of Pilar. Situation that is based on the theory of Caroll (1999), which builds a CSR model composed of four dimensions: the economic dimension, the legal dimension, the ethical dimension and the philanthropic dimension. From this basis, the categories of analysis that guide the study are defined. A descriptive, observational level methodology will be used with a qualitative approach only and according to the time that the research will be developed, it is prospective crosssectional. The population is made up of 12 accounting firms, legally established in the Municipality of Pilar. As a data collection technique, the self-assessment guide will be used, being, therefore, a self-administered questionnaire with binary answers (yes / no) and comments on the questions posed. The questionnaire corresponds to a self-application guide developed by ADEC-ETHOS called CSR 1.0 indicators. validated in Paraguay by the ADEC. The results find incipient initiatives not planned or established as organizational policies of accounting firms, which requires a research-action approach to reverse this situation towards a more socially responsible culture with clients, the community and with itself as an organization.
\end{abstract}

Keywords: Corporate Social; Responsibility; accounting studies.

Artículo recibido: 15 enero 2022 Aceptado para publicación: 08 febrero 2022 Correspondencia: torresnorimir@gmail.com Conflictos de Interés: Ninguna que declarar 


\section{INTRODUCCIÓN}

La Responsabilidad Social Empresarial (RSE) se ha convertido en uno de los temas que más se abordan en el mundo académico y empresarial por su impacto en lo económico, social y medio ambiental, pues los estudios demuestran que se puede ser socialmente responsable, mientras que se genera riqueza (Cancino del Castillo y Morales Parragué, 2008).

Pero ¿qué significa ser una empresa socialmente responsable?, si bien no existe una definición única sobre la RSE, la investigación acuña la definición descripta en la norma ISO 26000 (ISO, 2010) que define la responsabilidad social como la responsabilidad de una organización ante los impactos que sus decisiones y actividades ocasionan en la sociedad y el medioambiente, mediante un comportamiento ético y transparente.

\section{Este comportamiento debe ser expresado desde siete dimensiones fundamentales:}

1)gobernanza de la organización,

2) derechos humanos,

3) prácticas laborales,

4) medio ambiente,

5) prácticas justas de operación,

6) asuntos de consumidores,

7) participación activa y desarrollo de la comunidad.

Así también, la Teoría de Carroll (1999) que sustenta esta investigación permite definir que existen cuatro tipos principales de responsabilidades que debe asumir una empresa $u$ organización, como ser: económicas, legales, éticas y discrecionales. Para el autor, para que una empresa se pueda relacionar efectivamente con la RSE necesita tener una definición y entendimiento básico del significado e impacto del fenómeno.

En lo que respecta a la contabilidad, los estudios han demostrado el rol importante del contador en las acciones de RSE, de allí surgen la contabilidad ambiental y social. (Rodríguez, 2008).

La Contabilidad como una disciplina, debería contribuir al aumento de la responsabilidad social a través de la estructuración de sistemas de información contable que permitan efectuar un seguimiento adecuado del manejo de los recursos y de los impactos sociales de las organizaciones hacia adentro y hacia el contexto. (Rodriguez de Ramírez, 2004). 
Por ende, la formación integral y responsabilidad social del Contador Público depende ya de los principios previamente inculcados en el seno familiar, dentro de la sociedad donde se desenvuelve y en la universidad donde se forme, quienes son los responsables de implementar las mejores estrategias de educación superior para lanzar al mercado laborales, los mejores profesionales que promuevan ante la sociedad rectitud y ética entre otros valores, logrando así construir un sistema correcto y competitivo entre las empresas (Alfaro, 2018).

Estudios a nivel Latinoamérica, demuestran que la RSE está siendo fuertemente aplicadas en países como Brasil, Argentina, Chile, entre otros (Ramírez, 2009), no así en Paraguay, donde es un tema rechazado por el congreso según la última Resolución N²254 /06 (Congreso Nacional del Paraguay, 2016).

Si bien la RSE es un tema pendiente en Paraguay, organismos nacionales como la ADEC, entidades financieras, empresas y universidades empiezan a mostrar su compromiso con el ambiente y la sociedad, a la par que generar competitividad para generar mayor riqueza. Esto demuestra que, pese a la renuencia del estado en implementar políticas de RSE, existe una conciencia social que está abriendo camino a la construcción de una sociedad de consumo cada vez más responsable.

Principalmente, por que adoptar buenas prácticas de responsabilidad social ha demostrado impactar positivamente en un desarrollo local más sustentable y sostenible. Además, para la empresa, se ha convertido en un elemento importante de ventaja competitiva, y componente diferenciador entre los competidores (Gómez García, et, al 2017).

Desde esta perspectiva, varios autores sostienen que la RSE brinda unos contiguos efectos favorecedores para la empresa, tanto para el entorno interno de la empresa, como en la relación con sus intereses externos, al igual que beneficios tangible e intangible, que son evidenciables a corto y a largo plazo a través de estudios de evaluación cuantitativa y cualitativa (Valdez, Hernández y Jiménez, 2021: De Castro Chacón, 2021).

Tal es el caso de investigaciones desarrollada por Jimenez (2019), donde halla, en sus estudios llevados a cabo en las pymes de la ciudad de Pilar, que el nulo involucramiento de las empresas en cuestiones políticas, cívicas, problemáticas sociales, actividades sociales e incipientes liderazgo e influencia social, solo procuran evitar situaciones que involucren o favorezcan a agentes del poder público. Ninguna de las empresas patrocina 
programas públicos o privados de becas escolares, tampoco conciencia a sus empleados sobre la importancia de participar en las administraciones gubernamentales y de fiscalización.

Estos datos muestran que aún son incipientes y en muchos de los casos nulas las acciones de RSE en el ámbito empresarial a nivel local.

El mismo autor, más adelante estudió al público interno y su responsabilidad social empresarial dentro de las mismas medianas empresas de la ciudad de Pilar. Los resultados vuelven a mostrar resultados negativos, pues se halla nulas acciones de RSE, las empresas aplican deficientes políticas de remuneración, incluso con salarios por debajo del mínimo vigente por ley, y desconocimiento respecto a la satisfacción laboral de los empleados. (Jimenez, 2019).

Mas adelante, el mismo autor realiza una radiografía de la responsabilidad social empresarial y la situación de los grandes contribuyentes del sector comercial de la ciudad de Pilar. Los resultados demostraron que no hay actividades de promoción acerca de la RSE a nivel local, por ende, existe poco conocimiento acerca del tema, derivando en una cultura de baja prioridad para accionar al respecto, pese a que los encuestados consideran que las empresas poseen el dinero y el talento humano necesario para participar en acciones sociales (Jiménez, 2019).

El mismo autor también abordó en otra investigación la Responsabilidad social empresarial de las medianas empresas con la comunidad pilarense, desde una perspectiva cualitativa, dando como resultado que aún es ínfima la actuación en cuanto a RSE por parte de las medianas empresas pilarenses, al punto que, en la mayoría de los casos, dentro de sus organizaciones, ni siquiera se han tratado estos temas con anterioridad (Jiménez, 2019).

Morell Jiménez (2020), también investigó posteriormente a las medianas empresas del sector comercial de la ciudad de Pilar y su responsabilidad social empresarial con los grupos de interés externos, dando como resultado que las mismas, presentan nulas características de Responsabilidad Social Empresarial. Por consiguiente, la investigación propone como herramienta de gestión empresarial una propuesta de implementación de políticas de RSE mediante acciones que involucren a todos los sectores que forman parte importante de la actividad empresarial. 
El estado del arte sobre la RSE a nivel local demuestra que aún falta un largo camino por recorrer para conseguir enfocar a las empresas hacia un camino socialmente responsable. Estas informaciones coinciden con lo percibido por los estudiantes de Contabilidad, donde estudio realizado por Morell Jiménez y Villalba Chamorro (2020), concluyen que en la ciudad de Pilar se desarrolla un nivel intermedio de Responsabilidad Social y Desarrollo Sustentable, desde la percepción de los estudiantes universitarios de contabilidad. Dicho nivel se traduce en acciones mínimas aceptables de desarrollo, por tal razón, el panorama actual, desde las primeras impresiones de los estudiantes, motiva a abordar mejoras en cuanto a las dimensiones; estructura económica, agentes económicos e innovación, infraestructura, servicios y accesibilidad, calidad de vida y RRHH, cohesión social, organizaciones e identidad territorial, medio ambiente y recursos naturales y organizaciones. Ante esta realidad, los autores sugieren potenciar una cultura amigable, de manera a despertar una actitud reflexiva y fomentar, a través de ellas, la adopción de acciones socialmente responsables y sustentables.

Ante estas nulas acciones de RSE, se puede tomar el rol del contador como potencial líder en el fomento de una cultura de responsabilidad social (Ávila, 2021), ya que, para Reyes y Leal (2019), el contador público desempeña un papel esencial en la relación de la contabilidad con la responsabilidad social empresarial, primeramente por la función del contador público en la elaboración de informes contables y además, porque le corresponde el análisis y seguimiento de los recursos invertidos en proyectos de índole social, protección del medio ambiente y cambios tecnológicos.

Otro aspecto que puede dar una pauta de que los contadores pueden desarrollar acciones de RSE, es que el perfil de egreso de los mismos señala que el contador egresado, tomando el caso de la Universidad Nacional de Pilar, es capaz de proponer estrategias de desarrollo de la empresa observando la ética profesional, la responsabilidad social, el desarrollo económico y social sustentable del país preservando el medio ambiente (Facultad de Ciencias Contables, Administrativas y Económicas, 2021), coincidiendo el mismo perfil de egreso con otras universidades que ofertan la carrera de contaduría pública nacional en la ciudad de Pilar y que lanzan anualmente número importantes de contadores públicos nacionales al mercado laboral pilarense.

De toda la revisión bibliográfica analizada, surge algunas interrogantes ¿Los contadores públicos de la ciudad de Pilar son socialmente responsables?, de estas interrogantes 
surgen otras más específicas; ¿Cuáles son las prácticas de RSE que implementan los estudios contables? ¿En qué dimensión (social, ambiental, económico) cumplen con acciones de responsabilidad sociales?

Para responder a las interrogantes, se estableció como principal objetivo de investigación identificar las prácticas de responsabilidad social empresarial que vienen implementándose en los estudios contables de la ciudad de Pilar, año 2021.

\section{MATERIALES Y MÉTODOS}

La investigación sigue dos momentos de estudio, un primer momento que consta de una revisión bibliográfica del estado del arte de la temática de estudio y de la elección de la teoría que regirá la investigación.

De dicho análisis se elige la teoría de Caroll (1999), que construye un modelo de RSC compuesto por cuatro dimensiones: la dimensión económica, la dimensión legal, la dimensión ética y la dimensión filantrópica. De esta base se definen las categorías de análisis que direccionan el estudio.

La investigación adopta una metodología de nivel descriptivo, de diseño observacional con enfoque cualitativo y de corte trasversal prospectivo. De la misma se realiza una descripción holística de las prácticas de responsabilidad social empresarial que vienen implementándose en los estudios contables de la ciudad de Pilar, departamento de Ñeembucú en la República del Paraguay.

Es decir, la investigación cualitativa trata de identificar la naturaleza profunda de las realidades, su sistema de relaciones, su estructura dinámica, a través de registros narrativos (Rodríguez, 2015).

El universo de estudio lo conforman contadores públicos que trabajan de manera independiente en sus respectivos estudios contables en la ciudad de Pilar. Basados en el registro de Patente Comercial de la Municipalidad de Pilar, se encuentran con patente activo 12 estudios contables, la misma cantidad fueron utilizada como población de estudio. No se recurrió a una muestra representativa por ser una población poco numerosa y accesible de alcanzar.

Como técnica de recolección de datos se utilizó una entrevista semiestructurada. Como instrumento se empleó la guía de auto aplicación desarrollado por ADEC-ETHOS denominado indicadores de RSE 1.0. validado en Paraguay por la ADEC. 
La misma consta de 7 Dimensiones de autoevaluación. La investigación utilizó sólo 3 dimensiones que se adecua al contexto de los estudios contables, como ser: 1) RSE con el Medio Ambiente, 2) RSE con los consumidores y clientes y 3) RSE con la comunidad. Esta guía representa la entrevista semiestructurada de autoadministración con respuestas binarias (si/no) y opciones de comentarios abiertos respecto a las respuestas.

Éstas se identifican en el cuadro que representa las categorías de análisis y sus dimensiones:

Tabla 1. Categorías de análisis

\begin{tabular}{|c|c|c|}
\hline Categorías de & Dimensiones & Indicadores \\
\hline \multirow[t]{2}{*}{ Medio ambiente } & $\begin{array}{l}\text { Responsabilidad frente a } \\
\text { futuras generaciones }\end{array}$ & $\begin{array}{l}\text { - Compromiso con el mejoramiento } \\
\text { de la calidad ambiental } \\
\text { - Educación y concienciación } \\
\text { ambiental }\end{array}$ \\
\hline & $\begin{array}{l}\text { Gerenciamiento del } \\
\text { Impacto ambiental }\end{array}$ & $\begin{array}{l}\text { - Impacto sobre el medio ambiente } \\
\text { - Sustentabilidad de la economía } \\
\text { - Minimización de insumos }\end{array}$ \\
\hline $\begin{array}{l}\text { Consumidores y } \\
\text { Clientes }\end{array}$ & $\begin{array}{l}\text { Dimensión social del } \\
\text { consumo }\end{array}$ & $\begin{array}{l}\text { - Política de comunicación comercial } \\
\text { - Excelencia en la atención } \\
\text { - Conocimiento y Gerenciamiento de } \\
\text { los daños potenciales de productos y } \\
\text { servicios }\end{array}$ \\
\hline \multirow{2}{*}{ Comunidad } & $\begin{array}{l}\text { Relación con la } \\
\text { comunidad local }\end{array}$ & $\begin{array}{l}\text { - Impacto de la empresa en la } \\
\text { comunidad } \\
\text { - Relación con organizaciones locales }\end{array}$ \\
\hline & Acción Social & $\begin{array}{l}\text { - Financiamiento de la Acción Social } \\
\text { - Involucramiento con la acción } \\
\text { social }\end{array}$ \\
\hline
\end{tabular}

Fuente: elaboración propia en base a indicadores de RSE 1.0.

Para el análisis de los datos se recurrió a la utilización dela técnica deductiva explicadas por las 3 categorías que fueron analizaron en la investigación (medio ambiente, comunidad, consumidores y clientes). Cada respuesta fue resumida en términos de 
coincidencia o divergencia para poder comprender las acciones de RSE de los estudios contables de la ciudad de Pilar. Estas categorías y citas se representan en una descripción de resultados. A partir de los dichos hallazgos, se generalizaron los resultados y se exponen en forma de resumen detallando las partes más significativas de los descubrimientos.

\section{RESULTADOS Y DISCUSIÓN}

\section{Categoría de análisis 1. Medio ambiente}

Respecto al indicador de compromiso con la mejora de la calidad ambiental, los 12 contadores señalaron que no cuentan con una política ambiental formal, de conocimiento de todos los empleados, y que conste en el código de conducta y/o en la declaración de valores de la empresa. No obstante, 8/12 suele participar con frecuencia en comités/consejos locales o regionales para discutir la cuestión ambiental con el gobierno y la comunidad.

Igualmente, 8/12 contribuye a la preservación de la biodiversidad por medio de políticas específicas y/o proyecto (s) de conservación de áreas protegidas y/o programa de protección a través de organismos sin ánimo de lucro ubicados en la zona y que llevan adelante programas ambientales con miras a proteger el medio ambiente, principalmente los humedales y la flora y fauna de la región.

Si bien, dicha política no forma parte directa de sus respectivos estudios contables, estos contadores apoyan a los organismos que sí tienen como eje organizacional políticas de protección, conservación del medio ambiente, así como política explícita de no utilización de materiales e insumos provenientes de la explotación ilegal de recursos naturales (como madera, productos forestales no madereros, animales, etc.).

En cuanto a la Educación y Concientización Ambiental, sólo 2/12 contadores desarrollan periódicamente campañas internas de reducción del consumo de agua y de energía en sus respectivos estudios contables, el resto no señala indicios de acciones responsables con el agua y la energía eléctrica.

Por el contrario, no se evidencia estudio contable alguno, que desarrolla periódicamente campañas internas de educación con base en las tres r's: Reducción, Reutilización y Reciclaje. Puesto que los 12 entrevistados no tienen como hábitos estos tipos de prácticas en sus procesos comerciales y de servicios. 
Donde solo 2/12 profesionales contadores desarrollan periódicamente campañas internas de educación para el consumo consciente en sus respectivas organizaciones con sus empleados, pero su enfoque no es con miras sustentables si no con intereses económicos con el fin de reducir el gasto por energía eléctrica.

Respecto al Gerenciamiento del impacto ambiental, las acciones de los 12 contadores son nulos, pues los mismos no brinda a sus consumidores y clientes información detallada sobre daños ambientales resultantes del uso y del destino final de sus productos. Esto al menos en los casos de empresas que trabajen con productos o servicios que representan un riesgo, tanto para el medio ambiente, como para la comunidad.

No obstante, resalta como aspecto positivo que 8/12 contadores debate con los empleados, consumidores y clientes, proveedores y la comunidad sobre los impactos ambientales causados por sus productos o servicios. Además, 10/12 prioriza la contratación de proveedores que comprobadamente tengan buena conducta ambiental.

Por el contrario, los 12 estudios contables carecen de certificaciones ambientales como la ISO 14001, FSC, etc., así mismo, todos ellos, no implementan políticas de Sustentabilidad de la Economía Forestal.

Independientemente a las políticas, se rescata como aspecto positivo que 9/12 incentiva a sus proveedores a buscar la certificación ambiental, 11/12 prioriza y apoya a proveedores contratados en la búsqueda de la sustentabilidad forestal, y 8/12 busca la minimización de entradas y salidas de insumos como gestión cero papeles, elegir productos tecnológicos con eficiencia energética, utilizar productos de bajo consumo, entre otras.

También se rescata como aspecto positivo que 10/012 estudios contables realizan dentro de la empresa la reducción del consumo de agua y 11/12 realiza la reducción de la generación de residuos sólidos.

Por el contrario, es un tema pendiente para los 12 contadores de la ciudad de Pilar sobre las iniciativas para el uso de fuentes de energía renovable, y de las acciones de control de la polución causada por vehículos propios y de terceros a su servicio.

Estos hallazgos coinciden con lo identificado por Morell (2019:2020) en las empresas de la ciudad de Pilar y pone a urgente consideración lo que señala el informe de las Naciones unidas (2021), "el mundo no puede mantener nuestra tasa de uso y abuso para siempre, y 
es imperativo que aceptemos los cambios en los estilos de vida y los medios de vida necesarios para lograr los objetivos de 2030” (p.5).

\section{Categoría de análisis 2. Consumidores y Clientes}

La Responsabilidad social empresarial relacionados con la Política de Comunicación Comercial que implementan los estudios contables representa una de las dimensiones mejor desarrollas en este sector de la sociedad, puesto que los 12/12 de los contadores actúa rigurosamente de acuerdo con la legislación de defensa del consumidor. Todos ellos (12/12) tienen una política formal de comunicación alineada con sus valores y principios, 10/12 señalan poseer conciencia de su papel en la formación de valores y estándares de consumo y comportamiento de la sociedad.

Si bien para los 12/12 estas estrategias no están establecidas como política de comunicación comercial, como tampoco consta en el código de conducta y/o declaración de valores de su empresa. Se rescata que los 12/12 de contadores brindan una excelente calidad de la atención. Esto se debe a que para los 11/12 de los estudios contables, cuentan con una política y normas de relación con los clientes y consumidores que sí constan en el código de conducta y/o declaración de valores dela empresa.

En cuanto a la excelencia, en la calidad de atención a los consumidores y clientes, los 10/12 estudios contables promueven la capacitación continua de sus profesionales de atención al cliente para establecer una relación ética y de respeto de los derechos del consumidor. También, 9/12 entrena e incentiva a sus profesionales de atención al cliente a reconocer fallas y a actuar con rapidez y autonomía en la resolución de problemas, al igual que, 12/12 tiene una política explícita de no soborno para obtener la decisión de servicios y 11/12 posee una política formal de protección de la privacidad y/o un sistema de gestión de las informaciones privadas del consumidor, cliente o usuario.

Así también, resalta como aspecto positivo de responsabilidad social empresarial, el hecho de que 12/12 de los contadores con estudios contables informa al cliente el propósito de la solicitud de informaciones personales antes de hacerla. Al igual que, 11/12 solicita solamente informaciones personales relevantes y no más de la necesaria para los objetivos declarados y 12/12 poseen una política que permite al consumidor, cliente o usuario, la inclusión, alteración y exclusión de sus datos del banco de información de la empresa. 
Entre otras acciones de RSE por parte de los contadores de la ciudad de Pilar, resalta que 9/12 de ellos, llevan actualmente la Contabilidad ambiental de alguna empresa, principalmente las medianas empresas comerciales y algunas grandes empresas del rubro de construcción y servicios eléctricos.

También, 8/12 contadores desarrolla el Balance social de empresas u organizaciones, principalmente en organizaciones no gubernamentales que reciben donaciones a nivel internacionales para actividades de contenidos social, educativo y ambiental, y misma cantidad de estudios contables (8/12), elabora instrumentos de información con contenido de RSE para informes de licitaciones público-privadas, que respondan a los objetivos de desarrollo sostenible (ODS) establecido como parte del plan 2030 (Naciones Unidas, 2015).

Estos resultados coinciden con los hallazgos de Jimenez (2019), en empresas de la ciudad de Pilar, y pone de manifiesto la necesidad de tomar el liderazgo, desde la profesión contable, para diseñar políticas y estrategias de RSE que se alineen a los valores de la organización y que compartan dichas políticas con las empresas donde prestar servicios contables, coincidiendo con numerosos autores (Rodríguez, 2008; Valdez, Hernández y Jiménez, 2021: De Castro Chacón, 2021) sobre la importancia del rol del contador público en la búsqueda de la sostenibilidad de las empresas a través de su compromiso con la RSE con el medio ambiente, los clientes y consumidores y con el medio ambiente y como promotor, para asumir su parcela social para contribuir a manifestar aquella información de transcendencia social que verdaderamente refleje la RSC de las empresas aportando su carácter riguroso, concreto y contribuyendo a dar un paso más en la evolución de este proceso informativo (Herrera Pérez, 2020).

\section{Categoría de análisis 3. Comunidad}

La dimensión de RSE de los estudios contables con la comunidad representan importantes iniciativas, puesto que 12/12 de los contadores reconoce a la comunidad en la que está presente como parte interesada importante en sus procesos decisorios, 10/12 participa activamente de la discusión de problemas comunitarios y de la búsqueda de soluciones, 11/12 tiene prácticas de compra y de inversiones para contribuir con el desarrollo socioeconómico de la comunidad en la que está presente y 12/12 concientiza y capacita a sus empleados para que respeten los valores, conocimiento y prácticas tradicionales de la comunidad donde actúa. 
Por el contrario, se evidencia en los 11/12 estudios contables, incipientes acciones respecto a establecer políticas de relación con la comunidad de su entorno contempladas en su código de conducta y/o en la declaración de principios.

En relación a las Relaciones con Organizaciones Locales, los 12 estudios contables analizados conocen superficialmente sus actividades y responde puntualmente a pedidos eventuales de apoyo, 7/12 apoya varias entidades con donaciones, financiamiento e implementación de proyectos, divulgando experiencias exitosas y 10/12 participa en la elaboración e implantación de proyectos conjuntos con entidades locales, manteniendo asociaciones de largo plazo y capacitando a los liderazgos involucrados.

Así mismo, se observa que 10/12 estudios contables actúan en asociación con entidades locales buscando influenciar políticas públicas, estableciendo alianzas y participando de diversas redes para maximizar su contribución con el desarrollo local y 6/12 realizan en la comunidad, en conjunto con organizaciones locales, campañas educacionales y/o de interés público.

En cuanto al Financiamiento de la Acción Social, se encuentra incipientes acciones de RSE por parte de los contadores públicos, puesto que solo 2/12 estudios contables de la ciudad incluyen a la acción social y a sus responsables en el proceso general de planificación estratégica. Ninguno de ellos posee mecanismos para estimular a proveedores, accionistas y otras partes interesadas a hacer donaciones, tampoco utilizan especialistas en el ciclo de planificación, monitoreo y evaluación de su acción social.

Los 12 estudios contables no planean su acción social buscando maximizar su impacto a largo plazo, tampoco optimizan el impacto de su acción social fomentando recursos de otras empresas u organizaciones privadas y/o la participación de órganos públicos y no cuentan con un procedimiento de consulta periódica a los beneficiarios de su acción social, monitoreándola por medio de indicadores de desempeño.

Mientras que, en la dimensión de Involucramiento con la Acción Social, se destaca acciones de RSE con la comunidad de 8/12 estudios contables, a través de donaciones de productos y recursos financieros, como ceder instalaciones, movilizar el trabajo voluntario de sus empleados y/o desarrollar proyectos sociales propios. También 8/12 divulga internamente los proyectos que apoya y desarrolla, ofreciendo oportunidades de trabajo voluntario y estimulando la participación de los empleados. 
Estos datos coinciden con los resultados Morell (2020), ya que, si bien no tienen políticas específicas de RSE con la comunidad, realizan aportes económicos a pedido de las instituciones o personas interesadas y de esta manera contribuyen con responsabilidad social las demandas de la sociedad.

\section{CONCLUSIONES}

La Responsabilidad social empresarial en los contadores públicos se caracteriza por aisladas acciones en torno a iniciativas de RSE con los consumidores y clientes, con la dimensión social del consumo y con la comunidad a través de la relación con la comunidad local y algunas iniciativas no planificadas de acción social.

Éstas son consideradas incipientes para lo que conlleva ser una organización socialmente responsable según la teoría de Carroll (1999), y más cuando se trata de profesionales contadores, que tienen una relación directa con las empresas de los diferentes rubros que operan en la sociedad (Guibert Alva, 2013).

Esto llama la atención y abre un espacio para una reflexión socioambiental y económico más profunda, desde el punto de vista filantrópico, teniendo en cuenta que la RSE no es una obligación según ley en Paraguay, no obstante, la falta de presencia de los contadores en la lucha de los objetivos de desarrollo sostenible, demuestra el menos cabo de conciencia social y la falta de responsabilidad de los contadores con el entorno interno y externo en donde se desarrolla la profesión.

No obstante, pese a la falta de políticas bien establecidas que asumen la responsabilidad social empresarial de los estudios contables, resalta la intención, en términos filantrópicos, así como de misión y visión organizacional, la preocupación que sienten por las acciones medioambientales que buscan conservar y proteger al medio ambiente, frente a los efectos resultados de la producción de bienes y servicios de las empresas locales.

Los resultados obtenidos hasta aquí, concuerdan con lo que, ya investigadores como Morell (2019;2020), y Villalba Chamorro (2019; 2020), explicaban sobre el entorno interno y externo de la responsabilidad social y prácticas sustentables existentes en las empresas y en el municipio de la ciudad de Pilar.

El estado del arte, así como la presente investigación, demuestra que, en los sectores del tejido empresarial a nivel local, hay incipientes indicios de asumir prácticas de RSE con la comunidad, los clientes y el medio ambiente. Por ello se sugiere, ante tan preocupante 
contexto, proyectar una investigación-acción que permita a fomentar, concienciar, educar e instruir hacia la importancia de la adopción de prácticas de responsabilidad social y sustentabilidad en todo el proceso de la producción, y comercialización de bienes y servicios y que dichas acciones formen parte de las políticas organizacionales y de la planificación estratégica de cada estudio contable. Esto de manera a construir una comunidad modelo más limpia, más sostenible y sustentable. Al mismo tiempo de generar competitividad en el mercado.

Los informes medioambientales de las Naciones Unidas (2021) hablan de una afectación inminente de los recursos naturales, por ende, antes de que esto se empieza a notarse más de cerca, es inaprensible tomar acciones a priori y encaminar a la comunidad pilarense hacia una búsqueda permanente de responsabilidad social teniendo a los contadores como líderes en la incursión de estos valores y que compartan con las empresas, proveedores y clientes con quienes trabajan.

Pero la pregunta es ¿Cómo lograrlo?, se sugiere seguir el modelo diseñado por Morell Jiménez (2020, p.99) titulado "Modelo de responsabilidad social empresarial (RSE) para medianas empresas y que se encuentra ajustado al contexto local de la ciudad de Pilar". En su dimensión medioambiente, cliente y consumidores, y comunidad.

\section{LISTA DE REFERENCIA}

Asociación de Empresarios Cristianos (ADEC). http://www.adec.org.py/programasrse.php, consultado el 25 de enero de 2019.

Ávila, N. (2021). Responsabilidad social del contador público desde su proceso de formación para fortalecer la gestión contable de microempresarios en la localidad de suba rincón Bogotá. Colombia (Doctoral dissertation, Corporación Universitaria Minuto de Dios). Consultado de https://repository.uniminuto.edu/handle/10656/12363

Alfaro, A. C.(2017). La responsabilidad social del contador público en el campo económico y social en Colombia. Revista Brújula Digital, pp.1-9. Consultado de https://core.ac.uk/download/pdf/304920315.pdf

Cancino del Castillo, C., \& Morales Parragué, M. (2008). Responsabilidad social empresarial.

Carroll AB. Responsabilidad social empresarial: evolución de un constructo definitorio. Empresa y sociedad. 1999; 38 (3): 268-295. doi: 10.1177 / 000765039903800303 
De Castro Chacón, A. M., Díaz Nieto, A. M., \& Martínez Patiño, L. L. (2021). La Gestión Tributaria en CI J\&C SAS en provecho de los beneficios fiscales en el marco de responsabilidad social y la sostenibilidad.

Facultad de Ciencias Contables, Administrativas y Económicas (2021). Perfil de egreso del Contador. Consultado de https://contables.unp.edu.py/contaduria/

Gómez García, J. Vázquez E. y Cuervo M. (2017) La responsabilidad social empresarial: un fenómeno en evolución, en Políticas Públicas y Renovación Social en el Siglo XXI. ( $1^{\text {a }}$ ed.) Grupo Editorial Hess: México. pp. 227-258

Guibert Alva, G. M. (2013). El rol del contador público y su responsabilidad ética ante la sociedad. Consultado

de https://repositorioacademico.usmp.edu.pe/handle/20.500.12727/568

Congreso Nacional del Paraguay (2016). Consultado de http://odd.senado.gov.py/archivos/file/MHCD\%20Nro\%201739.pdf

ISO (2010). ISO 26000:2010 — Guidance on social responsibility Disponible en: http://www.iso.org/iso/catalogue detail?csnumber=42546. Consultado 26 de octubre 2021

Jimenez, E. D. M. (2019). Análisis de responsabilidad social empresarial (RSE) de medianas empresas en Pilar, Paraguay. Brazilian Journal of Development, 5(11), 22958-22969.

Jimenez, E. D. M. (2019). El público interno y responsabilidad social empresarial (RSE) de las medianas empresas de la ciudad de Pilar. Brazilian Journal of Development, 5(12), 28123-28138.

Jiménez, E. D. M. (2019). Responsabilidad social empresarial de las medianas empresas con la comunidad Pilarense. RInCE-Revista de Investigaciones del Departamento de Ciencias Económicas de La Universidad Nacional de la Matanza.

Jiménez, E. D. M. (2019). Responsabilidad Social Empresarial: una radiografía sobre la situación de los grandes contribuyentes del sector comercial de la Ciudad de Pilar. Revista Internacional de Investigación en Ciencias Sociales, 15(2).

Herrera Pérez, G. S. (2020). El Contador Público ambiental: precepto del futuro inherente a la RSE.

Morell Jiménez, E. D. (2020). Las medianas empresas del sector comercial de la ciudad de Pilar y su responsabilidad social empresarial con los grupos de interés externos. 
Morell Jiménez, E. D., \& Villalba Chamorro, A. A. (2020). Responsabilidad Social y Desarrollo Sustentable de la ciudad de Pilar; Una mirada desde la percepción de los estudiantes universitarios . Ciencia Latina Revista Científica Multidisciplinar, 4(2), 413-430. https://doi.org/10.37811/cl_rcm.v4i2.88

Naciones Unidas (22/mayo/2021). Cambio climático y medioambiente. Consultado de https://news.un.org/es/story/2021/05/1492352

Naciones Unidas (2015). Objetivos del Desarrollo Sostenible ODS. Consultado de https://www.un.org/sustainabledevelopment/es/objetivos-de-desarrollosostenible/

Ramírez, J. B. B. (2009). Fundamentos económicos y políticos del concepto de responsabilidad social empresarial en Latinoamérica: en debate. Revista Tendencias \& Retos, (14), 15-31.

Reyes, P. \& Leal, R. (2019). Responsabilidad Social Corporativa desde la contabilidad: Un mapeo sistemático de la literatura para Colombia. Apuntes contables, (24), 179-193.

https://doi.org/10.18601/16577175.n24.11

Rodriguez De Ramírez, M. D. C. (2004). Contabilidad y responsabilidad social en búsqueda de respuestas para una tendencia en vías de consolidación. Contabilidad Y Auditoría, (20), 22 Págs. Recuperado a partir de http://ojs.econ.uba.ar/index.php/Contyaudit/article/view/134

Rodríguez, D. H. (2008). La responsabilidad social empresarial en el contexto de la contabilidad social. Económicas CUC, 29(1), 185-192.

Rodríguez, E. M. R. (2015). Comprensión teórica y proceso metodológico de la investigación cualitativa. In Crescendo, 6(2), 169-183.

Valdez, M. Á. J., Hernández, C. A. J., \& Jiménez, S. O. (2021). Los beneficios de la responsabilidad social empresarial: una revisión literaria. Tiempo y economía, $8(2)$. 